\title{
The Effects of Feldenkrais Classes on the Health and Function of an Ageing Australian Sample: A Pilot Study
}

\author{
Susan Hillier ${ }^{*}$, , Louise Porter ${ }^{1}$, Kate Jackson ${ }^{2}$ and John Petkov ${ }^{3}$ \\ ${ }^{I}$ Centre for Allied Health Evidence, University of South Australia (City East), North Tec, Adelaide, SA 5000, Australia \\ ${ }^{2} 47$ Gladstone Rd, Mile End SA 5031, Australia \\ ${ }^{3}$ Centre for Regional Engagement, University of South Australia, Wireless Road West, Mount Gambier SA 5290, \\ Australia
}

\begin{abstract}
Participation in regular physical activity has a variety of health benefits including increased levels of function and independence for people who are ageing. The inclusion of motor learning principles into exercise programs is proposed to increase functional benefits. The presence of these principles in the Feldenkrais Method (FM) suggests this may be a beneficial program for the ageing population.
\end{abstract}

Objective: A proof of concept study was conducted to determine the effectiveness of an eight week movement class based on the FM when compared to a generic balance class.

Method: A pseudo-randomized controlled pilot study was conducted in an Australian community based organization of healthy people post-retirement, $\mathrm{n}=22$. Self-perceived health and functional status were measured by the Short-Form 36 (SF-36) and Patient-Specific Functional Scale (PSFS) respectively. Objective functional assessment, by a blinded assessor, included the Timed Up and Go Test (TUGT), Functional Reach Test (FRT), Single Leg Stance time (SLS) and Walk on Floor Eyes Closed (WOFEC) measures.

Results: There was a significant time effect for all measures except the WOFEC. Post hoc analysis demonstrated significant improvements for both the FM and generic groups in the SF-36, PSFS and FRT and for the FM group only in the SLS test.

Conclusions: Classes based on the FM are effective in improving health and functional measures in a healthy ageing population, equally so with the generic class. Considerations for future research include a randomized controlled trial in a rehabilitation setting, with a larger sample size and appropriate measures to detect relevant change in functional levels.

Keywords: Movement rehabilitation, Feldenkrais method, ageing, balance, motor learning.

\section{INTRODUCTION}

The health of the ageing population is of increasing concern - as a growing, heterogeneous group they can present with a diverse range of health needs, thus presenting a challenge for health care providers. Physical activity is considered to be a major component of any balanced healthy lifestyle in any age group. Participation in regular exercise provides a range of health benefits including decreased mortality [1], prevention of coronary heart disease [2] and control of diabetes [3], hypertension [4] and respiratory conditions [5]. Other benefits of physical activity of particular importance to the ageing population include a reduction in falls [6], decreased incidence of osteoporosis and osteoarthritis [7], decreased chance of stroke [8] and possible positive effects in Alzheimer's disease [9]. Physical activity also affords a number of gains in psychological wellbeing including a reported decrease in insomnia, stress and depression [5]. An added benefit may be the provision of

*Address correspondence to this author at the Centre for Allied Health Evidence, University of South Australia (City East), North Tec, Adelaide, SA 5000, Australia; Tel: 61-8-8302 2544; Fax: 61-8-8302 2766;

E-mail: susan.hillier@unisa.edu.au social interaction in a group setting, particularly where members can suffer from social isolation and depression [10].

An inadequate level of physical activity is reported to be one of the major preventable risk factors for injury and disease in the ageing Australian cohort [11]. Other risk factors include obesity and falls, which are also reported to be positively influenced by participation in regular physical activity $[12,13]$. The Medicare services provided to this age group are twice to three times the average number provided to the broader Australian population, which constitutes a considerable financial demand [11].

Several authors have reported that although resistance exercise programs are associated with muscle strength increases, there may be only minimal effects on functional capacity, thus for a broader effect, physical programs should include functional training using tasks from everyday life $[14,15]$. Behavioural and physiological motor control literature suggests such task-specific training incorporates direct skill practice (or as close to the target skills as possible) and the use of both motor and sensory strategies to introduce movement "problem solving" [16]. 
The presence of these motor control and motor learning principles in the Feldenkrais Method (FM) [17] suggests that this approach may be a beneficial program for the ageing population. The FM is a movement re-education or rehabilitation approach developed by Moshe Feldenkrais that aims to increase the learner's consciousness of how the body performs tasks and to then move more efficiently [18]. Wildman [18] suggests that this process includes increasing awareness of where tension and pain are present in the body, and how inefficient movement patterns may contribute to the discomfort. Previous studies investigating the effectiveness of the FM have involved a wide variety of ages and diagnoses thus providing a sound base for further investigation [17]. However this systematic review found a lack of research in the ageing cohort [17], a group in need of increased physical activity levels. In light of these findings there currently exists the need for quality research into the effectiveness of the FM and motor control principles to provide evidence for their use to improve or rehabilitate function in a healthy ageing cohort.

The current investigation tested the following hypotheses:

1. Participation in a class based on the FM will improve:

- $\quad$ self-perceived health and functional status

- functional mobility, and

- functional balance in a healthy ageing population.

2. Participation in a class based on the FM will improve the above areas to a greater extent when compared to a generic balance exercise class in the same population.

\section{MATERIALS AND METHODOLOGY}

The trial took the form of a pseudo-randomised, controlled clinical study with blinded pre- and post intervention assessment. The participant population was a healthy ageing cohort, attending the University of the Third Age (U3A). U3A is a community based organisation that provides education and health related classes and activities for people who have retired and has a large metropolitan membership thus allowing us access to a population with a wide range of older ages, co-morbidities and activity levels. Ethical approval was obtained from the U3A and the University of South Australia Human Ethics Committee, and the study was performed in accordance with the ethical standards laid down in the 1964 Declaration of Helsinki.

The inclusion criteria for the study were those required for membership of the U3A: 1) aged 55 years or over and 2) retired or semi-retired. Persons were excluded if they had a diagnosis of any health problems that were likely to be exacerbated by movement or participation in either of the study classes.

Recruitment was via an advertisement in the U3A newsletter. Potential participants were invited to contact the investigators by telephone for further information. If the willing persons met the criteria, they were asked to attend baseline testing where they also gave written, informed consent.
Twenty two subjects were recruited, aged between 60 and 88 years (mean $=71.1$ years, $\mathrm{SD}=8.7$ years) including 16 females and 6 males. These subjects formed equal groups of 11 for the FM and generic interventions. The FM class included subjects of mean age 71.9 years $(\mathrm{SD}=10.8$ years) with the mean age of the generic class 71.9 years $(\mathrm{SD}=6.45$ years).

\section{Pre- and Post Intervention Testing}

Subjective measures consisted of two questionnaires filled out prior to the objective measures. The Short-Form 36 (SF-36) [19] is a three-page general health questionnaire consisting of eleven questions requiring a tick-box answer. It is a multidimensional health measure assessing eight subsets of health: limitations in physical function, physical role, social function, emotional role, bodily pain, mental health, vitality and general health perceptions. This subjective measure has had extensive use reported in the literature and has been proven to be both valid and reliable [20]. The Patient-specific functional scale (PSFS) was developed as a standardised tool to measure changes in the performance of skills that are more meaningful to the individual [21]. It allows the participant to nominate and rate improvements in activities that are important to them rather than generic preselected activities as in other functional scales.

Objective assessment involved four tests chosen on the basis of being quick and simple to administer in a nonclinical setting, with proven reliability and validity. The Timed up and go test (TUG) [22] is a time-measured test to assess basic functional mobility in older adults [23]. In the test the subject starts in a seated position then rises from the chair, walks $3 \mathrm{~m}$, turns $180^{\circ}$, returns to the chair and sits down. The Functional reach test (FRT) is both a functional and balance test measuring how far one can reach forward without moving the feet [24]. The FRT is commonly used as a predictor of falls [16] and is also a reliable test of instability [24]. The Single leg stance (SLS) test requires the subject to stand on the preferred leg for as long as able while timed with a stopwatch, and is not subject to the same ceiling effects as the Berg balance scale [25]. The Walk on floor eyes closed test (WOF) was devised to identify imbalance across a variety of ages [26]. The measure was chosen as it is more demanding than more commonly employed measures of balance and presents a greater challenge to a healthy older population. The procedure involves the subject (with arms folded and eyes closed) taking as many steps as possible with a heel-toe pattern (tandem) without sidestepping (measured as number of steps with a maximum of 10).

The participants commenced baseline testing by completing the two questionnaires with assistance from the assessor as required, and were then tested in random order for all objective measures to avoid any carry-over effect or systematic fatigue. One experienced assessor (LP) conducted all tests and was blinded to group allocation at both time points. Intra-rater reliability testing was conducted for the TUG, FRT, and SLS measures. High Intraclass Correlation Coefficient (ICC) $(1,1)$ values were obtained (ICC $>0.97, \mathrm{p}$ $<0.001)$ indicating excellent consistency. The WOF measure was not included in the initial reliability assessment as it was added in as a replacement for the Berg Balance Scale, when 
our pilot/reliability study demonstrated that this latter scale had a ceiling effect.

The participants self-selected one of the two classes based on the time/day of the class that was most suitable for them, without being aware of the content of the time they had chosen. Both classes were held for one hour per week for eight weeks. The FM Awareness Through Movement ${ }^{\circledR}$ lessons were conducted by a trained Feldenkrais practitioner (KL). The lessons focused on awareness and general mobility (particularly trunk rotation, flexion and rolling) and functional activities including sitting in comfort, reaching and transition from lying on the floor. During the lessons, participants are encouraged to explore directed variations in movement strategies to achieve the overall activity. This requires them to pay attention to the relative ease of each option and make decisions on the most effective. The instructor encourages awareness of comfort and body position at all times rather than large efforts or repetitions. For example in rolling over, the instruction may be to pay attention to the action of the arm moving across the body, compared to the leg pushing on the floor, whether the eyes are leading the movement or whether the breath is being held or is independent of the motion.

The generic balance exercise class was based on an established exercise program - 'Falls \& Fractures: Beating the odds' [27]. This class was chosen for comparison as it was currently being offered at the U3A, included the appropriate generic content and could be taught by the same practitioner who was also an accredited trainer in these classes. The program consists of graded exercises for strengthening key muscle groups (adding weights); standing exercises (reducing support, closing eyes) and a "bone density component" (modified through to full jumps). Further details of both classes are available from the authors.

The participants were asked to return after the eight weeks of classes for re-assessment, which was conducted as for the baseline testing.

A Mixed Model (Random Coefficient Regression) was used with subjects designated as a random effect rather than a fixed effect. The main effects were analysed as time, group and group*time and where significance was found post hoc individual comparisons were performed with Bonferroni adjustment. One of the assumptions of the mixed model is that the random effects be normally distributed. For each analysis the intercepts for each subject were saved. Graphical analysis through the use of histograms showed that the distributions were very acceptable and thus this assumption was confirmed.

\section{RESULTS}

The groups were equal at baseline for age. Both intervention groups contained eight female and three male participants. Attendance at both classes was very good with 100 per cent attendance achieved by 18 of the 22 subjects across both groups. There were no drop outs from either group. No adverse events were reported and participant satisfaction was anecdotally high.

The pre and post intervention data (means and standard deviations) are summarized in Table 1. Analyses revealed significant time effects for all measures $(p<0.36$ to 0.000$)$ except the WOF $(p=0.056)$. There were no group or group*time effects. Therefore post hoc individual comparisons were performed to further examine the nature of the time effects. The results are reported in Table $\mathbf{1}$ as pvalues (with corrected values in brackets). This revealed that for the SF36, PSFS and FRT both groups improved significantly, though the FM group failed to reach significance if the corrected $\mathrm{p}$ value was adopted for the SF36. Given the nature of the data the corrected p-value was considered highly conservative. For the SLS test only the FM group reached significance ( $\mathrm{p}=0.016$ corrected). For the TUG and the WOF neither group individually reached significance.

Table 1. Individual Group Comparisons: Pre and Post Intervention Means (Standard Deviation) with Post Hoc Analyses Unadjusted p Values (and Bonferroni Corrected), $\mathbf{n = 1 1}$ in Each Group

\begin{tabular}{|c|c|c|c|c|}
\hline \multirow{2}{*}{ Measure } & Group & $\begin{array}{c}\text { Pre- } \\
\text { Intervention }\end{array}$ & $\begin{array}{c}\text { Post- } \\
\text { Intervention }\end{array}$ & $\begin{array}{c}\text { p-Value } \\
\text { (Corrected p) }\end{array}$ \\
\hline \hline \multirow{2}{*}{ SF-36* } & Feldenkrais & $69.7(22.42)$ & $80.49(13.39)$ & $\boldsymbol{0 . 0 3}(0.07)$ \\
\cline { 2 - 5 } & Generic & $66.34(15.69)$ & $79.98(12.27)$ & $\mathbf{0 . 0 1}(\mathbf{0 . 0 2})$ \\
\hline \multirow{2}{*}{ PSFS* } & Feldenkrais & $4.85(1.38)$ & $4.12(1.176)$ & $\mathbf{0 . 0 2 5}(\mathbf{0 . 0 5})$ \\
\cline { 2 - 5 } & Generic & $5.57(1.35)$ & $4.04(1.49)$ & $\mathbf{0 . 0 0 0 1 ( 0 . 0 0 0 1 )}$ \\
\hline \multirow{2}{*}{ TUG* } & Feldenkrais & $7.75(2.78)$ & $7.06(1.72)$ & $0.07(0.14)$ \\
\cline { 2 - 5 } & Generic & $7.39(1.18)$ & $6.92(0.88)$ & $0.215(0.43)$ \\
\hline \multirow{2}{*}{ FRT* } & Feldenkrais & $27.64(7.47)$ & $31.89(3.98)$ & $\mathbf{0 . 0 0 9}(\mathbf{0 . 0 1 9})$ \\
\cline { 2 - 5 } & Generic & $22.53(6.87)$ & $27.62(4.97)$ & $\mathbf{0 . 0 0 2}(\mathbf{0 . 0 5})$ \\
\hline \multirow{2}{*}{ SLS* } & Feldenkrais & $18.73(27.09)$ & $32.95(27.87)$ & $\mathbf{0 . 0 0 8}(\mathbf{0 . 0 1 6})$ \\
\cline { 2 - 5 } & Generic & $24.20(25.35)$ & $31.06(24.92)$ & $0.17(0.34)$ \\
\hline \multirow{2}{*}{ WOF } & Feldenkrais & $5.91(3.70)$ & $7.18(3.49)$ & $0.251(0.50)$ \\
\cline { 2 - 5 } & Generic & $7.64(4.63)$ & $9.45(4.39)$ & $0.107(0.215)$ \\
\hline
\end{tabular}

SF-36: Short Form 36 [19]; PSFS: Patient-specific functional scale [20]; TUG: Timed up and go test [22]; FRT: Functional reach test [24]; SLS: Single limb stance test [25]; WOF: walk on floor eyes closed test [26].

*Indicates significant time effect $\mathrm{p}<0.05 ;$ Bold/italicized indicates statistical significance.

\section{DISCUSSION}

The first aim of this study was to determine if an exercise class based on the FM improved self-perceived health and function in healthy ageing persons and also to ascertain if this class improved performance on objective functional measures. The data from this trial lend weight to these hypotheses with FM class participants improving significantly on both subjective measures of health and function and two of the four objective measures for balance and mobility functions.

The second aim was to determine if these improvements were greater than those afforded by an existing balance class. Literature based on motor control and motor learning has suggested that for functional improvements, an exercise class should include direct skill and problem solving practice with an emphasis on motor and sensory strategies [16]. Hence it was hypothesised that an intervention based on these components, such as the FM, would improve the above 
measures to a greater extent than a generic exercise class based on strengthening and balance principles. The results from this study offer some support for this hypothesis with a superior effect being found for the single leg stance test of balance in favour of the Feldenkrais group.

The tester was found to be consistent across all measures as indicated by high correlation values; hence this should not be a confounding factor for the study results. Some measures were found to be insensitive or inappropriate. For example the TUG was too easy for this healthy and very mobile population and, unexpectedly, the WOF was found to be too challenging with the majority of subjects unable to achieve greater than three steps in each trial. This occurrence was observed at both the baseline and post-intervention testing, hence our conclusion that the measure was indeed too difficult and inappropriate to detect significant change in this population. A pilot trial using the Berg Balance Scale [25] demonstrated this measure had a significant ceiling effect in this population - clearly finding the right level of responsiveness in balance measures is crucial in studies like these.

For the current study a true concealed and randomised allocation process would have been preferable. Unfortunately without subject remuneration, the participants were only willing to participate in the class that was held at the time/day of their preference. However we believe selfselection of class based on time and day preference offers a pseudo-randomisation in that there is nothing inherently significant about the day that would bias one group from the other in terms of ability to move/improve.

Shumway-Cook and Woolacott [16] suggest that any intervention including motor learning principles such as those claimed to be present in an FM intervention should improve function to a greater extent than a generic intervention. It remains to be confirmed that motor learning principles are inherent within the FM, but it was not the purpose of the current study to confirm the FM content.

The results of the current study are promising. Individual data suggest that the benefits of the FM may be more easily detected in those with greater movement restrictions. For example one participant in the FM group who was initially "significantly disabled" in the TUGT scores, achieved scores in the "normal" range post intervention. Therefore we would anticipate that the use of the FM in a subject group that is significantly more impaired at baseline should offer more obvious positive benefits, such as groups with arthritis or other motor impairments.

\section{CONCLUSION}

The current study certainly confirms that members of the 55 years and older cohort benefit from the provision of movement re/habilitation classes per se. We have shown that the Feldenkrais Method is safe, feasible and acceptable to this population. At this time there is not enough evidence to be confident about the specific benefits of the FM and whether the content reflects greater opportunities for functional improvement.

This information can be added to evidence that informs service providers and consumers in their choice of physical activity and rehabilitation classes. Given the growing numbers in the ageing cohort and funding constraints, such information is paramount to guide healthcare resources. The current study was a pilot as it is the first to investigate the functional benefits of the FM in the healthy ageing. The results are strongly supportive of further high quality research into the effectiveness of this rehabilitation method, using larger samples and responsive measures.

\section{ACKNOWLEDGEMENTS}

Participants from the University of the Third Age, Adelaide, Australia.

\section{CONFLICT OF INTEREST}

There are no conflicts of interest to declare in the conduct of this study, by the authors.

\section{REFERENCES}

[1] American College of Sports Medicine. Guidelines for exercise testing and prescription. $6^{\text {th }}$ ed. Philadelphia: Lea \& Febiger 2000.

[2] Tanasescu M, Leitzmann M, Rimm E, Willet W, Stampfer M, Hu F. Exercise type and intensity in relation to coronary heart disease in men. JAMA 2002; 288: 1994-2000.

[3] Castaneda C, Layne J, Munoz-Orians L, et al. A randomized controlled trial of resistance exercise training to improve glycaemic control in older adults with type 2 diabetes. Diabetes Care 2002; 25: 2335-41.

[4] Hagberg J, Park J, Brown M. The role of exercise training in the treatment of hypertension: an update. Sports Med 2000; 30: 193206.

[5] Hardman A, Stensel D. Physical activity and health: The evidence explained. New York: Routledge 2000.

[6] Howe TE, Rochester L, Jackson A, Banks P, Blair V. Exercise for improving balance in older people. Cochrane Database Syst Rev 2007; Issue 4. Art. No.: CD004963. DOI: 10.1002/14651858. CD004963.pub2.

[7] Suomi R, Collier D. Effects of arthritis exercise programs on functional fitness and perceived activities of daily living measures in older adults with arthritis. Arch Phys Med Rehabil 2003; 84: 1589-94.

[8] Halar E. Management of stroke risk factors during the process of rehabilitation: Secondary stroke prevention. Phys Med Rehabil Clin N Am 1999; 10: 839-56.

[9] Lindsay J, Laurin D, Verreault R, et al. Risk factors for Alzheimers disease and death: A prospective analysis from the Canadian study of health and ageing. Am J Epidemiol 2002; 156: 445-53.

[10] King A, Pruitt L, Phillips W, Oka R, Rodenberg A, Haskell W. Comparative effects of two physical activity programs on measured and perceived physical functioning and other health-related quality of life outcomes in older adults. J Gerontol 2000; 55A: M74-83.

[11] Australian Institute of Health and Welfare. Australia's Health 2004. cat no. AUS-44, Canberra: AIHW 2004.

[12] Giles-Corti B, Macintyre S, Clarkson J, Pikora T, Donovan D. Environmental and lifestyle associated with overweight and obesity in Perth, Australia. Am J Health Promot 2003; 18: 93-102.

[13] Steadman J, Donaldson N, Kalra L. A randomized controlled trial of an enhanced balance training program to improve mobility and reduce falls in the elderly patients. J Am Geriatr Soc 2003; 51: 84752.

[14] Refshauge K, Gass E. Musculoskeletal physiotherapy. Clinical science and evidence-based practice. London: ButterworthHeinemann 2004.

[15] Buchner DM, Larson EB, Wagner EH, Koepsell TD, de Lateur BJ. Evidence for a non-linear relationship between leg strength and gait speed. Age Ageing 1996; 25: 386-91.

[16] Shumway-Cook A, Woollacott M. Motor Control: Translating research into clinical practice. $3^{\text {rd }}$ ed. Baltimore: Lippincott, Williams and Wilkins 2007.

[17] Ernst E, Canter PH. The Feldenkrais Method: A systematic review of randomized clinical trials. Phys Med Rehab Kuror 2005; 15: 151-6. 
[18] Wildman F. Learning: The missing link in physical therapy. A radical view of the Feldenkrais Method. NZ J Physiother 1990; 18: 6-7.

[19] McCallum J. The SF-36® in an Australian sample: validating a new, generic health status measure. Aust J Public Health1995; 19: 160-6.

[20] Stewart AL, Hays RD, Ware JE. The MOS Short-form General Health Survey: reliability and validity in a patient population. Med Care 1998; 26: 724-35.

[21] Stratford P, Gill C, Westaway M, Binkley J. Assessing disability and change on individual patients: a report of a patient specific measure. Physiother Can 1995; 47: 258-62.

[22] Collen F. The measurement of standing balance after stroke. Physiother Theory Pract 1995; 11: 109-18. Aerospace Med 1968; 39: 277-82.

[27] Falls and fractures - Beating the odds: Your falls prevention exercise routine. Sydney: Osteoporosis Australia 1991.

Received: September 14, 2009

Revised: October 8, 2009

Accepted: March 4, 2010

(C) Hillier et al.; Licensee Bentham Open

This is an open access article licensed under the terms of the Creative Commons Attribution Non-Commercial License (http: //creativecommons.org/licenses/by$\mathrm{nc} / 3.0 /$ ) which permits unrestricted, non-commercial use, distribution and reproduction in any medium, provided the work is properly cited. 